\title{
INVENTÁRIOS QUANTITATIVOS DE ÁRVORES EM MATAS DE TERRA FIRME: HISTÓRICO COM ENFOQUE NA AMAZÔNIA BRASILEIRA
}

\author{
Alexandre Adalardo de OLIVEIRA
}

\begin{abstract}
RESUMO - Os principais inventários quantitativos de árvores na Amazônia são revisados, enfocando os estudos desenvolvidos no território brasileiro. Resumiu-se os principais resultados de cada trabalho e compilou-se os padrões obtidos para a comunidade arbórea da florestas de terra firme no sentido restrito. São discutidas a validade e eficiência da metodologia utilizada e sua importância para o entendimento da composição e estrutura dessas comunidades. Amostras de um hectare, utilizando a metodologia usual, tem fornecido informação insuficiente sobre a comunidade e não permitem a extrapolação dos resultados para áreas adjacentes. Pouco conhecimento tem sido gerado, a partir dessas parcelas, desde o estabelecimento dos padrões observados nos primeiros trabalhos. É sugerido um novo direcionamento dos estudos de inventários quantitativos de árvores na Amazônia brasileira.
\end{abstract}

Palavras-chave: Amazônia, inventário florestal, floresta de terra firme, estrutura da vegetação Quantitative Tree Inventories in Lowland Rain Forest: A Review for the Brazilian Amazon

ABSTRACT - The published results of quantitative tree inventories in Amazonia rain forest, especially those from the Brazilian Amazon, are reexamined. The principal results of each work are summarized, and patterns obtained from these are presented. The validity and efficiency of the methodology used to date and its importance for the understanding of structure and composition of these communities are discussed. A sample of one hectare, using the traditional methodology, provides insufficient information to extrapolate to adjacent areas. Little additional knowledge had been obtained from such plots since the establishment of patterns in the earliest studies. New directions for quantitative inventories of trees in the Brazilian Amazon are suggested.

Key-words: Amazon, Forest Inventories, Tropical Rain Forest, Vegetation Structure, Vegetation Composition

\section{HISTÓRICO}

O mais antigo inventário quantitativo de árvores de uma floresta neotropical foi desenvolvido por Davis \& Richards (1934), em Moraballi Creek na Guiana Inglesa. Para cada um dos cinco tipos de florestas primárias identificadas pelos autores foram inventariadas todas as árvores com mais de $10 \mathrm{~cm}$ de diâmetro à altura do peito (DAP) em 1,5 ha (122 x 122m), obtendo entre 59-94 espécies e 460-919 árvores para cada uma das parcelas. Os autores verificaram que cada formação florestal estava mais ou menos limitada a um tipo definido de solo e a uma estrutura peculiar, apesar da grande proporção de espécies encontradas em mais de um tipo de floresta. O método para identificação das espécies foi baseado no nome popular dado pelos auxiliares de campo nativos, que utilizavam essencialmente as características do tronco e do corte da madeira.

\footnotetext{
Universidade Paulista - UNIP, Lab. Botânica. Av. Paulista 900, 1²andar. 01310-100. São Paulo, SP. botanica@unip-objetivo.br
} 
Preocupado com o levantamento do potencial de madeira para a produção de carvão, Bastos (1948) publicou o primeiro estudo quantitativo de uma floresta amazônica no Brasil. Utilizou para isso uma amostragem de 1 ha dividida em parcelas descontínuas de $20 \mathrm{x}$ $50 \mathrm{~m}$, totalizando 891 árvores com mais de $15 \mathrm{~cm}$ de DAP. Para árvores com DAP $\geq 30 \mathrm{~cm}$, identificou 46 espécies entre 124 individuos.

Estimulados principalmente pelos estudos de Davis \& Richards $(1933,1934)$ e pela influência de Theodosius Dobzhansky em visita ao Brasil, pesquisadores do Instituto Agronômico do Norte (IAN) lideraram, na década de 50, estudos de inventários florestais na Amazônia, destacando-se os trabalhos de Black, Dobzhansky \& Pavan (1950) e Pires, Dobzhansky \& Black (1953).

Black et al. (1950) amostraram duas parcelas em matas de terra firme e uma em igapó, todas com 1 ha (100 $\mathrm{x} 100 \mathrm{~m})$. Próximo a Belém, estudaram a mata de igapó do Rio Guamá e a mata de terra firme de Mucambo obtendo respectivamente 60 espécies entre as 564 árvores e 87 espécies entre 423 árvores com DAP $\geq 10 \mathrm{~cm}$. Na mata de terra firme de Tefé encontraram 79 espécies entre 230 árvores com mais de $20 \mathrm{~cm}$ de DAP. Os autores notaram a baixa similaridade entre as parcelas e a alta porcentagem de espécies raras e, utilizando o método de oitavas de Preston (1948), concluíram que em 1 ha não são encontradas pouco mais do que metade das espécies existentes em uma associação e sugeriram não ser possivel generalizar conclusões sobre composição e estrutura baseadas em amostragens de parcelas pequenas.

Com o intuito de obter uma estimativa do número de espécies de árvores na comunidade de terra firme do Estado do Pará, Pires et al. (1953) inventariaram os indivíduos com DAP ${ }^{3} 10 \mathrm{~cm}$ em 3,5 ha na localidade de Castanha, totalizando 1432 árvores amostradas e 179 espécies (108 espécies para o primeiro hectare). Utilizando análises estatísticas entre o esperado para a distribuição das espécies ao acaso, segundo a distribuição de Poisson, e o encontrado nas parcelas, concluíram que a distribuição das espécies mais comuns era uniforme. Através do método de oitavas de Preston (1948) inferiram que cerca de 70 espécies que ocorriam na associação não foram amostradas no levantamento. Comparações com a parcela próxima a Belém estudada por Black et al. (1950) mostraram a grande diferença entre a composição e diversidade de áreas de terra firme relativamente próximas (Pires et al., 1953).

O trabalho de Cain et al. (1956) marca o início da utilização, na Amazônia, de parâmetros fitossociológicos como densidade, freqüência e valor de importância (Curtis \& Macintosh, 1951) e da metodologia de caracterização da vegetação de Raunkier (1934) utilizando formas de vida e tamanho de folha. Em área próxima ao estudo de Black et al. (1950), aqueles autores encontraram, em parcela de 2 ha ( 200 
$\mathrm{x} 100 \mathrm{~m}), 897$ árvores e 153 espécies com DAP $\geq 10 \mathrm{~cm}$, estando 144 espécies presentes no primeiro hectare amostrado. Os autores notaram que: 1) este tipo de floresta não apresenta dominância de uma ou poucas espécies como nas florestas de clima temperado; 2) existe certa dominância apenas ao nivel de família; 3) a maioria das espécies apresentam freqüência baixa; 4) a similaridade específica é muito baixa quando comparada com outras florestas de terra firme.

O primeiro inventário de árvores no médio rio Amazonas foi realizado por Robert Lechthaler em 1 ha de mata de terra firme na Reserva Florestal Ducke, no município de Manaus. Com o objetivo de verificar o potencial madeireiro na região, foram anotadas todas as árvores com mais de $8 \mathrm{~cm}$ de DAP e classificadas quanto à coloração da madeira. As árvores foram identificadas pelo nome popular e a metodologia utilizada para essa identificação não foi descrita; foram listados pelo menos 76 nomes populares entre os 735 individuos amostrados (Lechthaler, 1956).

A missão FAO na Amazônia, junto à Superintendência do Plano de Valorização Econômica da Amazônia (SPVEA), produziu vários levantamentos florestais que foram apresentados em forma de relatório ao governo brasileiro (Glerum, 1960; 1962a; 1962b; Glerum \& Smith, 1960; 1962; Heinsdijk, 1957; 1958a; 1958b; $1958 \mathrm{c} ; 1960 ; 1964 ; 1965 ; 1966$; Heinsdijk \& Bastos 1965; Bastos, 1960). Muitos deles tiveram uma divulgação muito restrita, sendo reunidos posteriormente em uma única publicação da Superintendência do Desenvolvimento da Amazônia (SUDAM, sucessora da SPVEA). A missão analisou pelo menos 1362 ha, onde as árvores com DAP $\geq 25 \mathrm{~cm}$ ou em alguns casos com mais de $45 \mathrm{~cm}$, foram medidas e identificadas por técnicos de campo especializados. Foram computadas 400 espécies entre 47 familias sendo Leguminosae, Lecythidaceae, Sapotaceae, Burseraceae, Lauraceae e Chrysobalanaceae as mais representativas. As florestas de terra firme na Amazônia foram separadas segundo dois tipos: Associação de Pouteria e Associação de Eschweilera-Pouteria, ambas divididas em 24 fácies ou variações segundo sua composição. Pela primeira e única vez semelhanças floristicas foram ressaltadas com a comparação entre 1 ha do rio Madeira com 111 indivíduos e 60 espécies e outro próximo a Belém com 133 individuos e 42 espécies. Estes inventários, distantes cerca de 1200 $\mathrm{km}$, apresentavam 22 espécies em comum, sendo mais da metade dos indivíduos em qualquer dos hectares pertencentes a espécies presentes às duas áreas. Qualitativamente a similaridade entre estas duas parcelas é pequena, sendo de $27,5 \%$ segundo o índice de Jaccard, ou seja, são menos semelhantes que o ressaltado pelos autores (SUDAM, 1974).

Na década de 60, Takeuchi estudou vários tipos de florestas na Amazônia utilizando perfis de 
vegetação para a análise da fisionomia. Em mata de terra firme destacaram-se o estudo próximo a Manaus (Takeuchi, 1960; 1961 ${ }^{1}$ ) e no alto Rio Negro (Takeuchi,1962). O tamanho de seus perfis variou muito e os dados de diversidade não foram apresentados já que as áreas amostradas, em média $500 \mathrm{~m}^{2}$, seriam pequenas para análise da densidade de espécies.

Rodrigues (1963) utilizando diâmetro de inclusão de $15 \mathrm{~cm}$ de DAP, encontrou na Serra do Navio, Amapá, 461 árvores e 96 espécies em 1,5 ha $(100 \times 150 \mathrm{~m})$. Em outra parcela de 1,1 ha $(100 \times 110 \mathrm{~m})$ computou 347 árvores e 84 espécies, sendo respectivamente 78 e 80 espécies para o primeiro hectare amostrado em cada uma das parcelas. $\mathrm{O}$ autor apresentou dados de cubagem de madeira, freqüência, densidade e diagramas de perfis de $100 \times 5 \mathrm{~m}$ para árvores acima de $3 \mathrm{~m}$ de altura, para cada uma das amostragens.

Grubb et al. (1963) estudaram fisionomia e estrutura de pequenas parcelas $\left(465 \mathrm{~m}^{2}\right)$ em floresta de terras baixas (matas de terra firme) e florestas montanas no Equador, encontrando, por parcela, entre 27-42 espécies e 42-101 árvores com mais de $6 \mathrm{~m}$ de altura. Os resultados obtidos contestaram a estratificação proposta para florestas tropicais e demonstraram uma similaridade na composição ao nivel de familias com a Hiléia Amazônica. Ao final, os autores propuseram uma nomenclatura um pouco diferenciada da de Richards (1952) para as formações tropicais, introduzindo o termo Florestas Tropicais Pluviais de Terras Baixas (Tropical Lowland Rain Forest).

Rodrigues (1967) buscando estudar a floresta ao longo da recém aberta estrada de Manaus- Itacoatiara, amostrou em 27 ha de parcelas árvores com DAP $\geq 25 \mathrm{~cm}$ e outros 27,5 ha para árvores com DAP $\geq 45 \mathrm{~cm}$. Encontrou as familias Leguminosae, Chrysobalanaceae, Lauraceae, Sapotaceae e Lecythidaceae como as mais representativas quanto ao número de espécie e de indivíduos.

Nos anos 70, o Projeto RADAMBRASIL elaborou o maior inventário da Amazônia brasileira investigando cerca de 2000 hectares, incluindo na amostragem todas as árvores com circunferência a altura do peito (CAP) maior ou igual a $100 \mathrm{~cm}$ (DAP $\geq 32 \mathrm{~cm})$. Contudo, estes dados foram apresentados em relatórios separados (RADAMBRASIL, 19681978) e somente na década de 90 uma síntese de parte dos resultados foi apresentada (Rollet, 1993). Esta compilação levou em conta os dados referentes às regiões de Boa Vista, Manaus e Purus (vols: 8, 17 e 18 RADAMBRASIL) que abrangem uma área de aproximadamente $840 \mathrm{mil} \mathrm{km}^{2}$ com cerca de $74 \%$ de áreas florestadas. Os 612 hectares analisados apresentavam 558 espécies, sendo as mais abundantes: Eschweilera odora ${ }^{2}$ (Poepp.) Miers (Lecythidaceae), Goupia glabra

\footnotetext{
'Esta publicação é a versão em inglês da anterior.

${ }^{2}$ Sinônimo de E.coriacea (DC.) Mori, em Mori \& Prance (1990).
} 
Aubl.(Celastraceae), Cariniana micrantha Ducke (Lecythidaceae), Licania membranacea Sagot ex Lanes (Chrysobalanaceae) e Pouteria guianensis Eyma (Sapotaceae) respectivamente (Rollet, 1993).

Ramos et al. (1972) levantaram o potencial madeireiro da região do Distrito Agropecuário da Zona Franca de Manaus utilizando métodos para obtenção de volume de madeira por hectare. Porém, os autores não deixaram claro qual o diâmetro de inclusão utilizado para as amostragens e não foram feitas as determinações das espécies amostradas, sendo portanto, este inventário, de pouca utilidade para discussão de composição, estrutura e diversidade da floresta Amazonia .

Pesquisadores ligados ao Instituto Nacional de Pesquisas da Amazônia (INPA) e Universidade Federal do Rio Grande do Sul (UFRGS) publicaram dois importantes trabalhos sobre fitossociologia de diferentes tipos de habitats na mata de terra firme próxima a Manaus (Prance et al., 1976; Porto et al. 1976). Prance et al. (1976) produziram o primeiro, e por muito tempo o único, estudo quantitativo da floresta de terra firme da região utilizando coletas para identificações ao nível específico. Foram encontradas em uma parcela de 1 ha $(125 \times 80 \mathrm{~m}) 179$ espécies e 350 árvores com DAP $\geq 15 \mathrm{~cm}$. Mais 56 espécies diferentes, com DAP entre 5$15 \mathrm{~cm}$, foram amostradas em uma subparcela de $200 \mathrm{~m}^{2}$, totalizando 235 espécies. Prance (1990) reapresentou os dados deste inventário computando Eschweilera odora $(=$ E. coriacea $) \mathrm{e}$ Scleronema micranthum (Ducke) Ducke (Bombacaceae) como as espécies com maior valor de importância (VIE) e, utilizando o valor de importância de familia (VIF) descrito por Mori et al. $(1983)^{3}$, determinou as principais familias para a área como sendo Lecythidaceae, Moraceae, Sapotaceae, Burseraceae, Caesalpiniaceae e Chrysobalanaceae.

Porto et al. (1976) publicaram o primeiro estudo de fitossociologia da "mata-de-baixio", vegetação ciliar de igarapés associada à mata de terra firme. Neste trabalho utilizaram critérios de inclusão diferenciados $(5,10$ e $30 \mathrm{~cm}$ de DAP) em diferentes áreas amostrais, totalizando 1 ha dividido em 10 parcelas descontinuas de $10 \times 100 \mathrm{~m}$. Os autores destacaram a unidade vegetacional desta formação e sua diferenciação floristica em relação à mata de terra firme adjacente e, seguindo a classificação de MuellerDombois \& Ellenberg (1974), denominaram a comunidade de VitexMicrandra. As espécies com maior densidade e freqüência foram Vitex sprucei Briq. (Verbenaceae) e Carapa guianensis Aubl. (Meliaceae) e as famílias mais representativas no dossel foram: Leguminosae, Myristicaceae, Sapotaceae, Meliaceae, Palmae, Euphorbiaceae, Annonaceae e Bombacaceae.

\section{Pires \& Prance (1977)}

\footnotetext{
${ }^{3}$ Aqui foram calculados os VIF do inventário de Prance et al. (1976), portanto esta é a primeira publicação a apresentar este tipo de descritor fitossociológico para uma floresta amazônica.
} 
apresentaram dados de distribuição vertical, horizontal e incremento no diâmetro das árvores de matas de terra firme, igapó e várzea. Todas as árvores com CAP $\geq 30 \mathrm{~cm}($ DAP $\geq 9,55 \mathrm{~cm})$ foram amostradas em uma área total de $15,5 \mathrm{ha}$, totalizando 8.996 indivíduos, 342 espécies e uma média de 580 áryores/ha. Deste total, 5,7 ha e 224 espécies foram registradas na mata de terra firme da reserva de Mucambo próximo a Belém; 4,7 ha e 180 espécies na mata de igapó da reserva de Catú, periférica a área de Mucambo, e 5,0 ha e 196 espécies na mata de várzea de Aurá. As espécies comuns entre as matas de terra firme e igapó totalizaram $43 \%$, entre mata de terra firme e várzea $38 \%$, entre várzea e igapó $40 \%$ e 83 espécies $(24 \%)$ ocorreram nos três tipos de florestas. Os autores não detectaram, nas áreas estudadas, qualquer tipo de estratificação como sugerido por Richards (1952) e predisseram que, apesar da ausência de espécies dominantes, em qualquer estudo na Amazônia com árvores com mais de 30 de CAP, de 5 a 15 espécies vão contribuir com cerca de $50 \%$ do número total de indivíduos presentes. Para os autores estas espécies devem ser consideradas como características da área, e baseados nos resultados discutem implicações para a conservação da Amazônia.

Em Altamira, Pará, foram relacionadas as plantas com pelo menos $30 \mathrm{~cm}$ de CAP em 1,5 ha de mata de terra firme sobre terra roxa, sendo encontradas 101 espécies (577 indivíduos) para uma parcela de 1 ha
(100 x 100) e 89 espécies (300 indivíduos) para a parcela de 0,5 ha. Foram utilizadas também subparcelas para a amostragem de árvores com CAP de 15 a $30 \mathrm{~cm}$ e para indivíduos com menos de $15 \mathrm{~cm}$ de CAP e mais de $2 \mathrm{~m}$ de altura (Dantas \& Müller, 1979).

Dantas et al.(1980) descreveram a fitossociologia da mata sobre latossolo amarelo em Capitão Poço, Pará, totalizando 121 espécies e 503 árvores com CAP $\geq 30 \mathrm{~cm}$ em 1 ha. As familias mais diversificadas foram: Leguminosae, Sapotaceae e Moraceae; as mais numerosas: Lecythidaceae e Burseraceae. A espécie com maior número de individuos foi Eschweilera odora $(=$ E. coriacea $)$.

Uhl \& Murphy investigaram a composição, estrutura e regeneração de uma mata de terra firme do sudeste da Venezuela. Encontraram, em matas maduras, 83 espécies e 744 árvores em 1 ha e em duas parcelas de 0,5 ha registraram 79 e 63 espécies entre 387 e 335 árvores respectivamente. A diversidade encontrada para uma amostragem de 0,5 ha em mata secundária foi muito semelhante, com 68 espécies entre 259 árvores. A composição da área foi pouco explorada, porém foram analisados alguns aspectos relacionados ao Valor de Importância Especifico (VIE) da comunidade. Para a parcela de 1 ha, 18 espécies contribuiram com $79,3 \%$ do total de valor de importância, sendo Licania sp a mais importante com $19,1 \%$ do VIE total. O VIE desta espécie variou muito entre os sítios de mata primária 
e os autores concluíram que uma espécie pode ser comum em uma amostra e rara ou ausente em outra próxima.

Ayres (1981), em seu estudo sobre comportamento e ecologia de primatas (Chiropotes albinasus e Chiropotes satanas), apresentou os dados de um levantamento de 8 ha em matas de terra firme com o objetivo de caracterizar o ambiente e levantar dados referentes às espécies utlizadas por estes macacos. Em sete parcelas de 1 ha em Aripuanã, Mato Grosso, encontrou uma média de 272,4 indivíduos por hectare e entre 28 e 33 familias para árvores com DAP $\geq .15$ $\mathrm{cm}$. As famílias dominantes ${ }^{4}$ foram: Leguminosae, Moraceae, Lauraceae e Myristicaceae, com variações dentro desta ordenação para os diferentes hectares. Utilizando o indice de similaridade entre familias de Bray \& Curtis (1957) encontrou valores entre $55-80 \%$ de similaridade entre quatro hectares a distâncias variadas, sugerindo para o autor que "dentro de uma pequena área de terra firme amazônica, a floresta é homogeneamente heterogênea". Em uma parcela de 1 ha em Aripuanã, as plantas com mais de $15 \mathrm{~cm}$ de DAP foram identificadas ao nível específico, sendo encontradas 123 espécies entre 266 árvores e Tetragastris altissima (Aubl.) Swart. (Burseraceae) a espécie com maior densidade. $\mathrm{O}$ valor de diversidade encontrado com o índice de Shannon na base 2 foi de 6,18 .
No Xingu, Campbell et al. (1986), utilizando amostragem em transectos e critério de inclusão de $\mathrm{DAP} \geq 10 \mathrm{~cm}$, investigaram três hectares $(3000 \times 10 \mathrm{~m})$ de mata de terra firme e meio hectare $(500 \times 10 \mathrm{~m})$ de várzea. Esta metodologia foi empregada porque os autores queriam "amostrar o maior número de espécies possível por unidade de área". Para a mata de terra firme foram encontradas 1420 indivíduos, 39 famílias e 265 espécies, sendo Cenostigma macrophyllum Tul. e Orbignya sp as espécies mais importantes (VIE) e Leguminosae, Palmae, Lecythidaceae, Moraceae e Bombacaceae as familias mais bem representadas. Para cada hectare foram encontradas $393,460 \mathrm{e}$ 567 árvores e 133, 118 e 162 espécies respectivamente. Apesar das duas espécies acima citadas terem sido computadas como as mais representativas para qualquer um dos hectares, a composição entre eles variou bastante. Apenas 40 espécies $(15 \%)$ foram registradas nos três hectares e a similaridade variou entre 26 $33 \%$ (índice de Jaccard) ${ }^{5}$ para cada dois hectares. Os autores concluiram que a alta diversidade da floresta era ocasionada pelo grande número de espécies raras que contribuíam com muito pouco do total de VIE da comunidade e que a Amazônia é um mosaico de diferentes tipos de florestas, não se devendo extrapolar os dados de caracterização e riqueza de espécies de pequenas amostragens para áreas mais amplas.

${ }^{4}$ Dominância é utilizada pelo autor no sentido de maior densidade ou maior número de individuos. 
Utilizando uma parcela retangular de 1 ha $(10 \times 1000 \mathrm{~m})$ em mata de terra firme na Bolivia, Boom (1986) descreveu a composição do estrato dominante (DAP $\geq$ $10 \mathrm{~cm}$ ) da floresta. Estes dados foram utilizados posteriormente para o estudo de etnobotânica quantitativa dos índios Chácobo (Boom, 1987). Neste levantamento foram encontrados 649 indivíduos pertencentes a 94 espécies, sendo Iryanthera juruensis Warb. (Myristicaceae), Pseudolmedia laevis (R.\&P.) Macbr. (Moraceae), Euterpe precatoria Mart. (Arecaceae), Pseudolmedia macrophylla Tréc. (Moraceae), Socratea exorrhiza (Mart.) H.Wendl. (Arecaceae) e Vochysia vismiifolia Spruce ex Warming (Vochysiaceae) as espécies com maior VIE e Moraceae, Myristicaceae, Palmae, Leguminosae, Melastomataceae e Cecropiaceae respectivamente as famílias com maior VIF. A diferença na importância das famílias, quando comparadas com outros trabalhos na Amazônia, a grande importância de Melastomataceae na amostragem e a baixa área basal da comunidade sugeriram ao autor que esta mata encontrava-se em uma fase de regeneração secundária de um distúrbio prévio.

Mori \& Boom (1987) e Mori et al. (1989) utilizaram o método de quadrante centrado (Cottam \& Curtis, 1956) e DAP de inclusão de $10 \mathrm{~cm}$ para descrever as vegetações de "La Fumée Mountain" na Guiana Francesa e Camaipi no Amapá, respectivamente.

$\mathrm{Na}$ Guiana Francesa, reserva de Saül, foram encontradas 295 espécies entre 800 árvores amostradas, sendo Burseraceae, Sapotaceae, Lecythidaceae, Mimosaceae e Caesalpiniaceae as famílias com maior VIF e Tetragastris altissima (Aubl.) Swart (Burseraceae), Quararibea turbinata Poir. (Bombacaceae), Virola michelii Heckel (Myristicaceae), Protium apiculatum Swart (Burseraceae), Couratari stellata A.C. Smith e Eschweilera coriacea (DC.) Mart. ex Berg (Lecythidaceae) as espécies com maior VIE. Utilizaram a relação entre o número de espécies de Lecythidaceae pelo número total de espécies na amostra para extrapolar o número de espécies esperado para reserva. Baseados na quantidade de espécies desta família coletadas por toda a reserva estimaram em 513 o número de espécies esperadas e concluíram que apenas $53 \%$ das espécies arbóreas teriam sido incluídas entre as 800 árvores amostradas (Mori \& Boom, 1987).

$\mathrm{Na}$ floresta de Camaipi no Amapá, foram computadas 205 espécies entre 1000 árvores, sendo: Geissospermum argenteum Woods. (Apocynaceae) a espécie com maior VIE, seguida de Tachigalia myrmecophila Ducke (Leguminosae), Tetragastris panamensis (Engl.) O. Kuntze (Burseraceae), Lecythis persistens Sagot. (Lecythidaceae) e Protium tenuifolium (Engl.) Engl. (Burseraceae). As famílias mais importantes foram: Apocynaceae,

\footnotetext{
${ }^{5}$ As similaridades apresentadas na publicação não condizem com os dados. Por algum motivo as espécies comuns aos três hectares foram excluídas do cálculo; os dados aqui apresentados foram recalculados levando em consideração estas espécies.
} 
Sapotaceae, Mimosaceae, Burseraceae e Lecythidaceae (Mori et al., 1989)
Absy et al. (1986/87) inventariaram seis hectares $(10 \times 1000$ $\mathrm{m})$, contíguos dois a dois, em floresta de terra firme na área de influência da estrada Cuiabá-Porto Velho (BR 364). Para árvores com DAP $\geq 10 \mathrm{~cm}$ foram encontradas entre 103-136 espécies por hectare de um total de 278 espécies e 2.235 indivíduos. Apesar da proximidade entre os hectares, as espécies mais importante para cada um deles variaram muito, sendo apenas duas comuns aos seis hectares entre o conjunto das 10 mais importantes de todos os hectares (Tetragastris altissima (Aubl.) Swart e Pseudolmedia laevis (R.\& P.) Macbr.). Entre as famílias com maior VIF a variação foi muito pequena, sendo Leguminosae, Moraceae, Sapotaceae, Lecythidaceae e Burseraceae as mais importantes, entre as seis mais, para qualquer um dos hectares.

Utilizando o método de quadrante centrado com inclusão de árvores com pelo menos $10 \mathrm{~cm}$ de DAP, na floresta amazônica do Equador, Balslev et al. (1987) compararam uma área de terra firme com uma mata de várzea adjacente no Parque Nacional de Yasuní. Na mata de terra firme, de um total de 804 árvores, encontraram 244 espécies e uma estimativa de 728 árvores e 228 espécies $^{6}$ por hectare sendo Iriartea deltoidea R.\&P. (Arecaceae), Rinorea $c f$. apiculata Hekking (Violaceae), Pseudolmedia laevis (R.\&P.) Macbr. (Moraceae), Cedrelinga catenaeformis Ducke (Leguminosae) e Cecropia sciadophylla Mart. (Cecropiaceae) as espécies com maior VIE e Arecaceae, Moraceae, Meliaceae, Mimosaceae, Caesalpiniaceae as familias com maior VIF.

No final da década de 80 , pesquisadores ligados ao Museu Paraense Emílio Goeldi (MPEG), desenvolveram muitos estudos sobre o componente arbóreo das florestas de terra firme na Serra de Carajás, Pará (Silva et al. 1986; Silva et al., 1987; Salomão et al. 1988; Silva \& Rosa, 1989) e em Rondônia (Maciel \& Lisboa, 1989; Lisboa, 1989; Salomão \& Lisboa, 1988).

Silva et al. (1986) registraram, para um transecto $(20 \times 500 \mathrm{~m}), 516$ árvores e 125 espécies com CAP $\geq 30$ $\mathrm{cm}$ e, considerando também o estrato arbustivo e herbáceo, encontraram Leguminosae, Moraceae, Rubiaceae, Bignoniaceae e Sapindaceae como as famílias mais diversificadas.

Silva et al. (1987) analisaram um transecto de 1 ha $(1000 \times 100 \mathrm{~m})$, registrando, para CAP $\geq 30 \mathrm{~cm}, 103$ espécies. A espécie com maior densidade foi Theobroma speciosa Willd. (Sterculiaceae), e Bertholletia excelsa M.\&B. (Lecythidaceae) a com maior área basal. As famílias com maior número de espécies foram respectivamente: Mimosaceae (13), Burseraceae (8), Moraceae (8), Caesalpiniaceae (8) e Papilionaceae (7) (Silva et al. 1987).

Utilizando 2 transectos de $500 \mathrm{x}$

\footnotetext{
${ }^{6}$ Segundo Mori (1987) e Mori et al. (1989) não se deve estimar a riqueza (spp/área) quando se usa o método do quadrante centrado pois este baseia-se na amostragem de indivíduos sendo área uma medida indiretamente relacionadas
} 
10 m, Salomão et al. (1988), investigaram também a mata de terra firme na região de Carajás, amostrando 484 indivíduos e 122 espécies de árvores com DAP $\geq 10 \mathrm{~cm}$ sendo Leguminosae, Sapotaceae, Vochysiaceae, Lauraceae e Melastomataceae as familias com maior VIF e Erisma uncinatum Warm. (Vochysiaceae) a espécie com maior VIE.

Silva \& Rosa (1989) levantaram os dados de duas áreas de terra firme sobre jazidas de cobre em Carajás com transectos de10x1000 m e inclusão de CAP $\geq 30 \mathrm{~cm}$. Encontraram 552 individuos e 119 espécies e 470 indivíduos 121 espécies para cada um dos transectos respectivamente, sendo Sapotaceae, Mimosaceae, Papilionaceae, Euphorbiaceae e Caeasalpiniaceae as familias com maior VIF para o primeiro hectare e Burseraceae, Mimosaceae, Moraceae, Lecythidaceae e Lauraceae as mais importantes no segundo hectare.

Em Rondônia, Salomão \& Lisboa (1988) e Maciel \& Lisboa (1989) estudaram a vegetação arbórea com amostragem de $500 \times 20 \mathrm{~m}$ para CAP $\geq 30 \mathrm{~cm}$ e pelo menos 10 subparcelas de $5 \times 1 \mathrm{~m}$ para a vegetação arbustivo-herbácea. Os primeiros encontraram, para o estrato arbóreo, 171 espécies entre 573 individuos, sendo Moraceae, Leguminosae, Burseraceae, Palmae e Sapotaceae as familias mais importantes e Tetragastris altissima (Aubl.) Swart (Burseraceae), seguida de Bertholletia excelsa H.\& B. (Lecythidaceae), as espécies com maior VIE (Salomão \& Lisboa, 1988). Maciel \& Lisboa (1989) anotaram 603 indivíduos pertencentes a 90 espécies e 33 familias no estrato arbóreo, sendo Theobroma cacao L. (Sterculiaceae) e Guarea kunthiana Adr. Juss. (Meliaceae) as espécies com maior VIE e Sterculiaceae, Meliaceae, Moraceae, Leguminosae e Palmae as famílias com maior VIF.

Também em Rondônia, Lisboa (1989) estudou a mata secundária levantando dados sobre todos os indivíduos $(\mathrm{n}=760)$ com $\mathrm{CAP} \geq 15$ $\mathrm{cm}$ em 0,5 ha $(500 \times 10 \mathrm{~m})$. As familias Leguminosae, Euphorbiaceae, Cochlospermaceae, Moraceae e Tiliaceae apresentaram maior VIF, sendo Cochlospermum orinoccense (H.B.K.) Steud.(Cochlospermaceae), Sapium marmieri Huber (Euphorbiaceae) e Inga edulis Mart.(Leguminosae) as espécies mais importantes entre as 113 encontradas.

Morellato \& Rosa (1991), com o objetivo de caracterizar os diferentes tipos de vegetação da Serra de Carajas, estabeleceram parcelas ao longo de transectos. Para o estrato arbóreo da floresta de terra firme madura (DAP $\geq$ $5 \mathrm{~cm}$ ) foram amostradas 100 espécies entre 437 indivíduos, sendo Erisma uncinatum, Aparisthium cordatum e Neea $s p$ as espécies ${ }^{7}$ que apresentaram maior VIE. Sâo apresentados também, os dados de composição e estrutura para os outros tipos de vegetação reconhecidos (Morellato \& Rosa, 1991).

Balée $(1986 ; 1987)$ realizou dois inventários em mata de terra firme com o objetivo de estudar a etnobotânica dos indios Ka'apor no Maranhão e Tembé no Pará, 
encontrando uma utilização, pelas comunidades indígenas, de $100 \%$ das espécies amostradas nos levantamentos. Na floresta pré-amazônica do Maranhão foram encontrados, em um transecto de $20 \times 500 \mathrm{~m}, 519$ individuos, 117 espécies de árvores e 6 espécies de cipós com DAP $\geq 10 \mathrm{~cm}$ (Balée, 1986). Na bacia do rio Gurupi, Pará, amostrou um lote de 1 ha $(10 \times 1000 \mathrm{~m}) \mathrm{com} 456$ árvores e 138 espécies, sendo Eschweilera sp (Lecythidaceae) seguida de Sagotia racemosa Baill. (Euphorbiaceae) as espécies com maior densidade (Balée, 1987).

Alwyn H. Gentry iniciou na década de 80 uma série de publicações relativas a padrões de diversidade de plantas neotropicais (Gentry 1982; 1986; 1988b; 1992; Gentry \& Emmons 1987). Em 1986, Gentry apresentou os dados de diversidade de duas parcelas de 0,1 ha na Colômbia com a impressionante média de 262 espécies com DAP $\geq 2,5 \mathrm{~cm}$. Em seu inventário em sete parcelas de 1 ha próximo a Iquitos, Peru, encontrou entre $283^{8}$ e 275 espécies de árvores com DAP $\geq 10 \mathrm{~cm}$ (Gentry, 1988a). Os dados foram comparados com outros inventários e o autor concluiu que a Amazônia Ocidental apresentava a maior alfa-diversidade de árvores do mundo, devido a seus solos relativamente mais férteis e a alta pluviosidade sem uma sazonalidade marcante (Gentry, 1988a). No mesmo ano publicou uma compilação e comparação dos dados de composição e diversidade de florestas de 87 estudos de vários autores em 25 paises diferentes (Gentry, 1988b).

Faber-Langendoen e Gentry (1991) descreveram a composição de uma floresta na região do Chocó na Colômbia, amostrando árvores com DAP $\geq 10 \mathrm{~cm}$ em parcelas de $1 \mathrm{ha}$ $(100 \times 100 \mathrm{~m})$ e 0,5 ha $(50 \times 100 \mathrm{~m})$ e indivíduos com DAP $\geq 2,5 \mathrm{~cm}$ em subamostras de 0,1 ha em cada uma das parcelas maiores. Foram encontradas, para a classe de árvores com DAP $\geq 10 \mathrm{~cm}, 252$ espécies para a amostragem de 1 ha e 154 espécies para a de 0,5 ha. As familias com maior VIF foram Arecaceae, Sapotaceae, Myristicaceae, Clusiaceae e Fabaceae, e as espécies ${ }^{9}$ mais importantes: Jessenia bataua, Socratea exorrhiza e Wettinia quinaria (Arecaceae) para a amostragem de 1 ha e Jessenia bataua, Otoba lehmannii (Myristicaceae) e Eschweilera panamensis (Lecythidaceae) para a parcela de 0,5 ha.

Salomão (1991) estudou a vegetação de mata de terra firme no município de Marabá, Pará. Todos os individuos com DAP $\geq 10 \mathrm{~cm}$ foram amostrados em seis transectos $(1000 \mathrm{x}$ $10 \mathrm{~m}$ ) contíguos dois a dois, sendo encontradas entre 104-109 espécies por hectare, de um total de 237 espécies amostradas. As familias mais importantes para a amostragem total foram: Euphorbiaceae, Burseraceae, Mimosaceae, Moraceae e

\footnotetext{
${ }^{7}$ Não foram apresentados os autores das espécies na publicação.

${ }^{8}$ Por muito tempo este foi o inventário quantitativo com o maior número de espécies amostradas por hectare, contabilizando 300 se levadas em consideração as lianas lenhosas.
} 
Lecythidaceae. Para quatro hectares Sagotia brachysepala (Muell. Arg.) R. Secco (Euphorbiaceae) foi a espécie mais importante e Tetragastris altissima (Aubl.) Swart (Burseraceae), a com maior VIE para os outros dois.

Com a intenção de comparar diferentes metodologias de amostragens em inventários florestais quantitativos, Korning et al. (1991) estabeleceram 1 ha $(100 \times 100 \mathrm{~m}) \mathrm{em}$ área adjacente ao estudo de Balslev et al. (1987) no Equador. Foram encontradas uma densidade de 734 individuos e 153 espécies por hectare, sendo Quararibea ochrocalyx (Schum.) Vischer (Bombacaceae), Jessenia bataua (Mart.) Burret ${ }^{10}$ (Arecaceae) e Virola elongata (Benth.) Warb. (Myristicaceae) as espécies com maior VIE e Bombacaceae, Arecaceae e Moraceae as famílias com maior VIF. Os autores observaram que a parcela quadrada amostrava menor riqueza, enquanto o transecto de ponto centrado incluiu uma maior variedade de habitats e, por conseqüência, maior riqueza de espécies. Concluiram que os dois métodos apontaram as tendência de dominância da floresta e que comparações de estrutura e diversidade só poderiam ser feitas quando baseadas no mesmo método de amostragem.

Silva et al. (1992) registraram, em um levantamento de quatro transectos $(10 \times 1000 \mathrm{~m})$ de mata na bacia do Juruá, 556 espécies (213-270 espécies/ha) e 3158 indivíduos com $\mathrm{DAP} \geq 10 \mathrm{~cm}$, uma das maiores alfadiversidade de árvores conhecida para a Amazônia brasileira até este momento e uma das maiores do mundo para este diâmetro de inclusão. As famílias que mostraram maior VIF entre os quatro hectares foram Chrysobalanaceae, Lecythidaceae, Leguminosae e Sapotaceae não necessariamente nesta ordem. Eschweilera odora Miers (Lecythidaceae) e Ragala sanguinolenta" Pierre (Sapotaceae); apresentaram maior VIE nos transectos 1 e 2 respectivamente e Eschweilera alba Kunth. (Lecythidaceae) foi a mais importante para os outros dois. Apareceram em segundo na classificação de VIE, Pourouma ovata Tréc. (Cecropiaceae) no transecto 1, Jessenia bataua (Mart.) Burret para o 2 e 3 e Eschweilera odora para o transecto 4.

Rankin-de-Merona et al. (1992) publicaram os dados preliminares do levantamento de 70 ha de mata de terra firme próximo a Manaus, dentro das reservas do Projeto Dinâmica Biológica de Fragmentos Florestais (PDBFF). Foram computadas 698 morfoespécies sendo as familias Lecythidaceae, Leguminosae, Sapotaceae e Burseraceae as mais abundantes em individuos e ricas em espécies.

Thompson et al. (1992) estudaram os fatores físicos, o

\footnotetext{
${ }^{9}$ Não foram apresentados os autores das espécies citadas na publicação.

${ }^{10}$ Sinônimo de Oenocarpus bataua Mart. segundo Henderson (1994).

"Sinônimo de Chrysophyllum sanguinolentum (Pierre) Baehni segundo Pennington (1990).
} 
componente estrutural da floresta e a química das folhas em seis parcelas com 0,25 ha cada em mata secundária tardia de terra firme na Ilha de Maracá, Roraima. Encontraram 630 árvores e 84 espécies com DAP $\geq 10$ $\mathrm{cm}$, sendo Moraceae e Sapotaceae as familias que apresentaram maior área basal.

$$
\text { Milliken et al. }
$$
apresentaram os dados preliminares do inventário de árvores de 1 ha de mata $(500 \times 20 \mathrm{~m})$ em seu estudo de etnobotânica na reserva dos índios Waimiri-Atroari no estado do Amazonas, posteriormente publicado de forma definitiva (Milliken, 1998). Foram encontradas, neste estudo, 200 espécies de árvores e 14 de lianas, sendo $81 \%$ das espécies de algum modo úteis para estes índios. As familias com maior número de indivíduos foram: Leguminosae, Lecythidaceae,

Burseraceae, Sapotaceae e Chrysobalanaceae, as espécies Clathrotropis macrocarpa Ducke (Leguminosae), Eschweilera coriacea, Protium hebetatum Daly, $P$. apiculatum Swart. (Burseraceae) e Oenocarpus bacaba Mart (Arecaceae).

$\mathrm{Na}$ Estação Científica "Ferreira Penna", em Caxiuanã, Pará, foram amostrados quatro hectares de mata de terra firme totalizando 2441 indivíduos de 338 espécies. O número de espécies para cada hectare variou de 147 a 196 e o número de indivíduos ficou entre 527-727, sendo as espécies mais abundantes: Laetia procera
Eichl. (Flacourtiaceae), Astrocaryum aculeatum G.F.W.Mey (Arecaceae), Goupia glabra Aubl. (Celastraceae) e Eschweilera coriacea. A hipótese de que haveria um gradiente de diversidade crescente no sentido Leste-Oeste, na Amazônia Oriental, não foi sustentada pela comparação entre os levantamentos deste estudo e de outros na região (Almeida et al., 1993).

Muniz et al. (1994a; 1994b) estudaram a composição e estrutura de mata de terra firme pré-amazônica remanescente da região de São Luis, Maranhão. Foram amostradas 50 parcelas de $10 \times 10 \mathrm{~m}$ contendo 410 indivíduos e 46 espécies arbóreas com $\mathrm{CAP} \geq 15 \mathrm{~cm}(\mathrm{DAP} \geq 4,77)$. Licania cf. incana Aubl., Guarea guidonia (L.) Sleumer e Copaifera langsdorffii Desf. foram as espécies que apresentaram maior valor de importância (VIE) na comunidade (Muniz et al., 1994b).

Dois hectares foram amostrados no noroeste da Guiana onde todas as árvores com DAP $\geq 10 \mathrm{~cm}$ congregaram 59 e 85 espécies e 493 e 504 individuos para cada um dos hectares respectivamente. A espécie mais importante em ambos hectares foi Eschweilera pedicellata ${ }^{12}$ (Lecythidaceae), sendo Aspidosperma excelsum (Apocynaceae) e Mora gonggrijpii (Caesalpiniaceae) as que aparecem em segundo lugar para o hectare 1 e 2 respectivamente. Esta última espécie, apesar de ser a segunda

\footnotetext{
${ }^{12}$ Não foram apresentadas os autores das espécies citadas na publicação.
} 
em importância para um dos hectares, foi totalmente ausente no outro (Comiskey et al., 1994).

Recentemente, Valencia et al. (1994) amostraram o hectare (100 x $100 \mathrm{~m}$ ) com maior alfa-diversidade de árvores do mundo, no Equador. Foram computadas 473 espécies com DAP $\geq$ $5 \mathrm{~cm}$ e 307 espécies com pelo menos $10 \mathrm{~cm}$ de DAP. Para esta última classe, Jessenia bataua ${ }^{12}$ (Arecaceae) e Eschweilera aff. coriacea (Lecythidaceae) foram as espécies com maior densidade e Leguminosae, Lauraceae, Sapotaceae, Moraceae e Burseraceae as familias mais diversas.

Em 1 ha de terra firme no municipio de Jenaro Herrera, Peru, foram amostradas 504 árvores e 227 espécies com DAP $\geq 10 \mathrm{~cm}$. Jessenia bataua (Mart.) Burret (Arecaceae), Eschweilera coriacea e Osteophloeum platyspermum (A.DC.) Warb. (Myristicaceae) foram as espécies que computaram maior VIE e Leguminosae, Sapotaceae e Moraceae as famílias com maior VIF (Spichiger et al. 1996).

Na Amazônia colombiana, Duivenvoorden $(1995,1996)$ utilizou 95 unidades amostrais de $0,1 \mathrm{ha}$, em áreas descontinuas, para o estudo de diferentes tipos de matas. As parcelas foram alocadas ao longo de um trecho do rio Caquetá, distanciadas nunca menos e geralmente mais do que 250 $\mathrm{m}$ uma das outras. Foram obtidos ao todo 1077 morfoespécies sendo 525 identificadas ao nivel de espécie. Nas 40 parcelas de terra firme foram computadas uma média de $38 \pm 1,4$ morfoespécies por parcela, $252 \pm 21$ espécies por hectare e um total de 668 morfoespécies. $\mathrm{O}$ estudo mostrou que a densidade de espécies correlacionase positivamente com altura do dossel e que a densidade de táxons em diferentes formações florestais apresenta relação com a qualidade do solo e hostilidade ambiental (Duivenvoorden, 1996).

Estudando as florestas da região do rio Urucu no Amazonas, Amaral (1996) encontrou uma alta beta diversidade, a maior já registrada para o Brasil. Em parcelas de 1ha (100x10 $\mathrm{m})$, pegando toda a transição entre matas de platô, encosta e baixio em cada uma, encontrou 253,269 e 322 espécies de árvores com mais de 10 $\mathrm{cm}$ de DAP para cada uma das amostras. Este estudo demonstra que há, além da alta diversidade pontual já reportada em outros estudos, também uma grande substituição de espécies quando tomamos um gradiente ambiental. É interessante notar que esta alta beta-diversidade, está também relacionada a uma diferença na estruturação da floresta que apresenta, no hectares mais diverso 769 individuos, uma densidade muito alta de árvores por hectare.

$\mathrm{Na}$ Amazônia Brasileira, próximo a Manaus, foram estabelecidas três parcelas de um hectare em floresta de terra firme em semelhantes condições edáficas e distando no máximo $400 \mathrm{~m}$. Foram computadas entre 280 a 285 espécies por hectare e um total de 513 espécies

\footnotetext{
${ }^{12}$ Não foram apresentadas os autores das espécies citadas na publicação.
} 
de árvores com DAP $\geq 10 \mathrm{~cm}$, uma das maiores densidades de espécies já relatada para florestas tropicais (Oliveira \& Mori, in press, Oliveira, 1997). A alfa diversidade foi relacionada a confluência de regiões fitogeográficas devido, provavelmente, a eventos de vicariância relativamente recentes (Oliveira \& Daly, in press; Oliveira, 1997). Os hectares apresentaram baixa similaridade sendo compartilhadas menos de $36 \%$ das espécies relacionadas a cada dois hectares. Eschweilera coriaceae (Lecythidaceae), Pouteria anomala (Pires) T.D.Penn. (Sapotaceae) e Scleronema micranthum (Bombacaceae) foram as espécies com maior VIE para cada um dos hectares respectivamente. Apenas $S$. micranthum, Protium hebetatum (Burseraceae) e Minquartia guianensis Aubl. (Olacaceae) aparecem entre as dez espécies com maior VIE nos três hectares, indicando a inconsistência da caracterização da comunidade utilizando este índice para amostras desta magnitude em florestas de terra firme (Oliveira, 1997).

No Parque Nacional do Jaú, Ferreira \& Prance (1998) analisaram quatro hectares descontínuos de floresta em terra firme onde todos os individuos com DAP $\geq 10 \mathrm{~cm}$ foram computados. A similaridade entre as amostras foi muito baixa, sendo apenas $40(13 \%)$ das 315 espécies amostradas comuns aos quatro hectares e apenas entre $24-44 \%$ comum a cada dois hectares. O número de espécies variou entre 137 a 168 e o número de individuos entre 639 a 713 , por hectare. As familias Burseraceae,
Leguminosae e Myristicaceae apresentaram a maior densidade de árvores em três dos hectares, não necessariamente nesta ordem, e Arecaceae, Chrysobalanaceae e Leguminosae as com maior densidade no quarto hectare. As espécies com maior abundância em relação ao número de indivíduos foram: Alexa grandiflora Ducke (Leguminosae) e Iryanthera laevis Markgr. (Mysristicaceae) para os hectares 1 e 3; Scleronema micranthum (Bombacaceae) e Protium grandifolium Engl. (Burseraceae) no hectare 2 e, Oenocarpus bacaba (Arecaceae) e Couepia obovata Ducke (Chrysobalanaceae) no hectare 4.

Utilizando os dados de inventários em larga escala nas Guyanas, Steege (1998) reconhece cinco regiões de florestas, sendo incluidas mais duas por informações adicionais, e através das análises estabelece estratégia para a delimitação de áreas de proteção natural. Foram analisados 1029 parcelas, totalizando 212 ha, e 15397 árvores com mais de $30,5 \mathrm{~cm}$ de DAP, sendo Caesalpiniaceae, Lecythidaceae, Fabaceae, Chrysobalanaceae, Sapotaceae, Mimosaceae e Lauraceae as famílias com maior densidade de indivíduos respectivamente.

\section{DISCUSSÃO}

Os inventários na Amazônia têm demonstrado que as matas de terra firme "senso strictu", ou seja as florestas de platô ou encosta em solo argiloso, apresentam alta diversidade, 
grande porcentagem de espécies com baixa densidade e baixa similaridade floristica entre parcelas próximas. Estes padrões foram estabelecidos muito cedo na história dos inventários florestais quantitativos (Davis \& Richard, 1934; Black et al., 1950; Pires et al., 1953; Cain et al., 1956), muito pouco tendo sido acrescentado desde então quanto a padrões estruturais e florísticos.

Comparações entre diferentes estudos foram dificultadas devido à grande variedade de metodologias empregadas, principalmente quanto à forma e tamanho da área amostral, e diâmetro de inclusão. Além disso, o grande esforço para a obtenção de boas coletas de material-botânicotestemunha e a complexidade na identificações destas, dificulta ainda mais comparações florísticas e estruturais ao nivel de espécie.

É difícil destacar espécies que, de um modo geral, apresentem grande importância na estrutura dessas florestas; sendo o mata-matá (Eschweilera coriacea, Lecythidaceae) a única que aparece com grande densidade em levantamentos por toda a Amazônia (Oliveira, 1997, Valencia et al., 1994; Rollet, 1993; Silva et al., 1992; Prance et al., 1976; Sudam, 1974; Cain et al.,1956; Pires et. al., 1953). Contudo, alguns padrões bem estabelecidos emergem das análises ao nivel de família onde, com raras exceções, entre as famílias com maior densidade e diversidade no dossel de matas primárias estão Leguminosae (sensu lato), Lecythidaceae, Sapotaceae,
Burseraceae, Chrysobalanaceae, Moraceae e Lauraceae.

Terbourgh e Andresen (1998), analisando dados de inventários de várias parcelas da Amazônia em terra firme e várzea, relata Arecaceae como sendo uma das familias melhor representadas, junto com Leguminosae. Em nosso levantamento, notamos que as palmeiras são predominantes em algumas florestas na transição da Amazônia com outros biomas, em ambientes perturbados, próximos a comunidades indígenas ou tradicionais, e em formações de floresta de baixio. Neste mesmo estudo Chrysobalanaceae é tratada como uma família de menor expressividade na comunidade de árvores da Amazônia o que também não parece estar de acordo com os dados aqui apresentados (Tab. 1), onde esta familia aparece entre as mais expressivas em grande parte dos inventários. Provavelmente estas diferenças são devidas principalmente a inclusão na análise de Terbourgh e Andresen (1998) de florestas periodicamente inundadas (igapó e várzeas) e pela ausência de amostras na região do médio curso do rio Amazonas, sendo a grande maioria das parcelas concentradas no extremo leste e oeste da Amazônia. Comparações e sinteses em níveis supra específicos devem ser estimuladas, principalmente na tentativa de buscar padrões florísticos de diferentes regiões e a classificação mais adequada de tipos de florestas, cuidando para representar igualmente todas as regiões da Amazônia e diferenciar as parcelas em florestas secundárias.

Alguns padrões de diversidade 
de árvores foram estabelecidos para as florestas de terra firme da Amazônia. Gentry (1988a) observou um gradiente de diversidade de árvores do leste para o oeste, com as parcelas mais próximas aos Andes apresentando maior riqueza (Gentry, 1988a; 1988b). O mesmo autor relacionou este fenômeno a fatores climáticos e edáficos, verificando que climas menos sazonais e solos relativamente mais ricos em nutrientes comportam maior diversidade de árvores (Gentry, 1988a). Outros autores relacionam a diversidade das parcelas à dinâmica natural de mortalidade de árvores (Phillips et al., 1994), contudo a sazonalidade climática parece ser $o$ fator melhor correlacionado (Gentry, 1992; Phillips et al., 1994; Clinnebell II et al.; 1995).

A Amazônia Oriental concentra grande número de inventários e todos eles apresentam menor riqueza do que os inventários mais a leste (Tab. 2) entretanto, parcelas no médio Amazonas apresentam densidades de espécies de árvores (DAP $\geq 10 \mathrm{~cm}$ ) muito próximas aquelas encontradas nos inventários da Amazônia Ocidental (Gentry, 1988a; Valencia et al., 1994; Oliveira, 1997; Oliveira \& Mori, no prelo), fugindo do padrão de gradientes longitudinal Leste-Oeste.

\section{CONCLUSÃO}

Até o momento, nota-se que, apesar do grande esforço já desprendido para se entender a estrutura e composição das florestas amazônicas, pouco ainda se sabe sobre padrões estruturais e diferenciações regionais. Uma sintese desses estudos se faz necessária, porém, mais do que isso é importante discutir o real valor das análises fitossociológicas em matas de terra firme baseadas em áreas de 1 ha, método que tem sido empregado como padrão de amostragem. Quais questões podem ser abordadas em amostras desta magnitude e até que ponto as conclusões podem ser estendidas para áreas adjacentes? Pode-se caracterizar a vegetação ou mesmo classificar as diferentes fácies das florestas, com esses tipo de amostra? Dados recentes têm demonstrado que os resultados de descritores de estrutura e composição obtidos à partir de amostras de um hectare não podem ser extrapolados para a floresta adjacente, mesmo para áreas muito próximas e em mesmas condições de topografia e solo (Oliveira, 1997). É necessário utilizar metodologias com maior eficiência e que permitam a extrapolação dos dados para um universo melhor definido e efetivamente maior do que $\mathrm{a}$ área amostrada. $\mathrm{O}$ desenho amostral deve ser planejado com cuidado e direcionado a questões bem definidas e que possibilitem o acesso a novas informações sobre essas comunidades.

Muitos pontos importantes relativos à ecologia de comunidade de árvores e sobre a diversificação nos trópicos começaram a ser levantados e esclarecidos a partir de inventários permanentes em áreas maiores, onde todos os individuos, mapeados e identificados, são acompanhados em estudos a médio e longo prazo 
Tabela 1. Principais familias e espécies botânicas em inventários de árvores em floresta de terra firme na Amazônia

\begin{tabular}{|c|c|c|c|}
\hline AUTOR & LOCALUDADE & FAMILIA & ESPECIE \\
\hline Oliveira, 1997 & Manaus, Brasil & $\begin{array}{l}\text { Sapo, Lecy, Legu, Burs, Laur, Chry, Mora, } \\
\text { Olac, Cecr }\end{array}$ & $\begin{array}{l}\text { Eschweilera coriacea, Pouteria anomala, } \\
\text { Scleronema micranthum }\end{array}$ \\
\hline Valencia et al, 1994 & Equador & $\begin{array}{l}\text { Legu, Laur, Sapo, Mora, Burs, Chry, Euph. } \\
\text { Myri, Cecr, Meli }\end{array}$ & Jessenia bataua, Eschweilera aff. cortacea \\
\hline Almeida ef al., 1993 & Caxiuanâ, Brasil & $\begin{array}{l}\text { Legu. Sapo, Mora. Laur, Burs. Chry, Anno, } \\
\text { Lecy, Mynt, Apoc. }\end{array}$ & Laetia procera Astrocaryum aculeatum \\
\hline Rankin-de-Merona ot at, 1992 & Manaus, Brasil & $\begin{array}{l}\text { Lecy, Leg, Sapo, Burs, Viol, Anno, Laur. } \\
\text { Mora, Chry, Euph. }\end{array}$ & $\begin{array}{l}\text { Micrandropsis scleroloxyon Protium of. } \\
\text { llewelynit }\end{array}$ \\
\hline Silva et al.. 1992 & Juruá. Brasil & $\begin{array}{l}\text { Legu, Sapo, Lecy, Chry, Mora, Laur, Myri. } \\
\text { Euph, Arec, Burs }\end{array}$ & Eschwellera alba Regala sanguinolenta \\
\hline Faber-Langendoen \& Gentry, 1991 & Chooó, Colombia & $\begin{array}{l}\text { Arec, Sapo, Myri, Clus, Faba, Burs, Lecy, } \\
\text { Mora }\end{array}$ & Jessenia bataua, Socratea exorrhiza \\
\hline Salomáo, 1991 & Marabá, Brasil & $\begin{array}{l}\text { Euph, Burs, Mimo, Mora, Lecy, Meli, Papi. } \\
\text { Anno, Caes, Laur }\end{array}$ & Sagotia brachysepala Tetragastris altissima \\
\hline Maciel \& Lisboa, 1989 & Rondônia, Brasil & $\begin{array}{l}\text { Ster, Meli, Mora, Legu, Arec, Sapo, Phyt. } \\
\text { Bomb, Flac, Poac }\end{array}$ & Theobroma cacau Guarea Kunthiana \\
\hline Mori et al., 1989 & Amapá, Brasil & $\begin{array}{l}\text { Apoc, Sapo, Mimo, Burs, Lecy, Caes, Chry. } \\
\text { Mela, Laur, Faba }\end{array}$ & $\begin{array}{l}\text { Geissospermum argenteum Tachigalia } \\
\text { myrmecophila }\end{array}$ \\
\hline Silva \& Rosa, 1989 & Carajés I, Brasill & $\begin{array}{l}\text { Sapo, Mimo, Papi, Euph, Caes, Laur, Mela, } \\
\text { Ruta, Sapi, Humi }\end{array}$ & Poecilanthe effusa Aparisthmium cordatum \\
\hline Silva \& Rosa, 1989 & Carajás II, Brasil & $\begin{array}{l}\text { Burs, Mimo, Mora, Lecy, Laur, Caes, Sapo, } \\
\text { Anno, Meli, Sapi }\end{array}$ & Tetragastris panamensis Protium apiculatum \\
\hline Salomâo \& Lisboa, 1988 & Ji-Paranà, Brasil & $\begin{array}{l}\text { Mora, Burs, Palm, Caes, Sapo, Lecy, Meli, } \\
\text { Papi, Bign, Mimo }\end{array}$ & Tetragastris attissima Bertholletia excelsa \\
\hline Salomào et at. 1988 & Carajás, Brasii & $\begin{array}{l}\text { Legu, Sapo, Voch, Laur, Mela, Nyct, Euph, } \\
\text { Myrt, Lecy, Meli }\end{array}$ & Erisma uncinatum Neea sp2 \\
\hline Balslev et al,., 1987 & Yasuni, Equador & $\begin{array}{l}\text { Palm, Mora, Meli, Mimo, Caes, Laur, Myri, } \\
\text { Lecy, Rubi, Euph }\end{array}$ & Iryartea deltoidea Rinorea cf. apiculata \\
\hline Mori \& Boom, 1987 & Guiana Francesa & $\begin{array}{l}\text { Burs, Sapo, Lecy, Mimo, Caes, Rubi, Euph, } \\
\text { Mora, Chry, Meli }\end{array}$ & Tetragastris altissima Quararibea turbinata \\
\hline Absy et al., 1986/87 & Janú I, Brasil & $\begin{array}{l}\text { Legu, Mora, Sapo, Lecy, Burs, Paim, Euph, } \\
\text { Viol }\end{array}$ & $\begin{array}{l}\text { Tetragastris altissima Tabebuia } \\
\text { avellannadeae }\end{array}$ \\
\hline Absy et al. $1986 / 87$ & Jarú II. Brasil & $\begin{array}{l}\text { Legu, Mora, Sapo, Lecy, Arec, Burs, Apoc, } \\
\text { Euph }\end{array}$ & Astronium lecointel, Aspidosperma oblongum \\
\hline Absy et al.,1986/87 & Ariquemes, Brasil & $\begin{array}{l}\text { Legu, Mora, Burs, Euph, Lecy, Sapo, Anac. } \\
\text { Arec }\end{array}$ & Macrolobium sp; Newtonia suaveolens \\
\hline Absy ef al., 1986/87 & Ariquemes, Brasil & $\begin{array}{l}\text { Legu, Mora, Lecy, Burs, Arec. Euph. Ster. } \\
\text { Sapo }\end{array}$ & Gallesia integrifolia: Macrolobium sp \\
\hline Absy et al. 1986/87 & Ariquemes, Brasil & $\begin{array}{l}\text { Legu, Mora, Lecy, Burs, Sapo, Euph, Myrl, } \\
\text { Bomb }\end{array}$ & $\begin{array}{l}\text { Couratant macrosperma; Totragastris } \\
\text { altissima }\end{array}$ \\
\hline Absy et al., 1986/87 & Ariquemes, Brasil & $\begin{array}{l}\text { Legu, Mora, Lecy, Sapo, Burs, Euph, } \\
\text { Bomb, Anac }\end{array}$ & Peltogyne catingae: Tetragastris altissima \\
\hline Boom, 1986 & Alto Ivon, Bolivia & $\begin{array}{l}\text { Mora, Myri, Arec, Legu, Mela, Cecr, Voch, } \\
\text { Anno, Chry, Rubi }\end{array}$ & Iryanthera juruensis; Pseudolmedia laevis \\
\hline Campbell et at. 1986 & Xingu, Brasil & $\begin{array}{l}\text { Legu, Arec, Lecy, Mora, Bomb, Meli, Ster: } \\
\text { Nyct, Chry, Sapo }\end{array}$ & Cenostigma macrophyla; Orbgnya sp1 \\
\hline Dantas et af, 1980 & Capităo Poço. Brasil & Lecy, Legu, Sapo, Mora & Eschweilera odora Theobroma subincanum \\
\hline Prance et al., 1976 & Manaus, Brasil & $\begin{array}{l}\text { Lecy, Mora, Sapo, Burs, Caes, Chry, Voch. } \\
\text { Laur, Mela, Humi }\end{array}$ & Eschwellera odora \\
\hline Rodrigues, 1963 & Amapá, Brasil & Legu, Sapo, Voch & Qualea ct. rosea; Pouteria virescens \\
\hline Cain et al., 1956 & Belém, Brasil & $\begin{array}{l}\text { Burs, Lecy, Voch, Legu, Sapo, Cela, } \\
\text { Myri, Ster, Anac, Clus, }\end{array}$ & Vochysia guianensis; Eschiweilera odora \\
\hline Pires et al., 1953 & Belém, Brasil & Lecy. Sapo, Legu & Eschweilera krukoviï; Eschweilera odora \\
\hline
\end{tabular}

Anac $=$ Anacardiaceae $, \mathrm{Apoc}=\mathrm{Apocynaceae}, \mathrm{Arec}=$ Arecaceae, Bomb=Bombacaceae, Burs $=$ Burseraceae, Caes $=$ Caesalpiniaceae, $\mathrm{Cecr}=$ Cecropiaceae, $\mathrm{Cela}=$ Celastraceae, $\mathrm{Chry}=\mathrm{Chrys}$ obalanaceae, Clus $=$ Clusiaceae, Euph= Euphorbiaceae, Flac $=$ Flacourtiaceae, Humi= Humiriaceae, Laur $=$ Lauraceae, Lecy $=$ Lecythidaceae, Legu $=$ Leguminosae sensu lato, Mela $=$ Melastomataceae, Meli= Meliaceae, Mimo = Mimosaceae, Mora $=$ Moraceae, Myri $=$ Myristicaceae, Nyct $=$ Nyctaginaceae, Olac $=$ Olacaceae, Papi $=$ Fabaceae sensu stricto, Phyt $=$ Phytolacaceae Poac $=$ Poaceae, Rubi= Rubiaceae, Sapi $=$ Sapindaceae, Sapo $=$ Sapotaceae, Ster $=$ Sterculiaceae, Viol= Violaceae, Voch $=$ Vochysiaceae 
Tabela 2. Principais estudos do componente arbóreo em florestas de terra firme da Amazônia e a riqueza encontrada

\begin{tabular}{|c|c|c|c|c|c|c|}
\hline Autor & Localidade & Amossta & $\begin{array}{l}\mathrm{DAP} \\
(\mathrm{cm}) \\
\end{array}$ & $N$ ind. & $\mathrm{N}^{0} \mathrm{sp}$ & $\mathrm{sp} / \mathrm{ha}$ \\
\hline Ferreira \& Prance, 1998 & Brasil, Jaú & $4(100 \times 100)$ & 10 & $639-713$ & 315 & $137-168$ \\
\hline Oliveira, 1997 & Brasil, Manaus & $3(100 \times 100)$ & 10 & 1916 & 513 & $280-285$ \\
\hline Dulivenvoorden, 1996 & Colómbia, Caquetá & $40(20 \times 50)$ & 10 & 2874 & 668 & $252 \pm 21$ \\
\hline Valencia et al., 1994 & Equador & $100 \times 100$ & 10 & 693 & 307 & 307 \\
\hline Almeida et al., 1993 & Brasil, Caxiuanã & $4(25 \times 400)$ & 10 & 2441 & 338 & $147-196$ \\
\hline Miliken et al., 1992 & Brasil, Maré & $20 \times 500$ & 10 & 643 & 201 & 201 \\
\hline Silva et al., 1992 & Brasil, Junuá & $4(10 \times 1000)$ & 10 & 3158 & 556 & $213-271$ \\
\hline Faber-Langendoen \& Gentry, 1991 & Colombia, Chocó & $100 \times 100$ & 10 & 675 & 258 & 258 \\
\hline Salomåa, 1991 & Brasil, Marabá & $3(20 \times 1000)$ & 10 & 3147 & 237 & $101-109$ \\
\hline Maciel \& Lisboa, 1989 & Brasil, Rondônia & 1 ha & 9,55 & 602 & 90 & 90 \\
\hline Mori et al.. 1989 & Brasil, Amapá & QC & 10 & 1000 & 205 & - \\
\hline Siva \& Rosa, 1989 & Brasil, Carajás & $2(10 \times 1000)$ & 9,55 & 1022 & $>121$ & $118-121$ \\
\hline Salomão \& Lisboa, 1988 & Brasil, Ji-Paraná & $500 \times 20$ & 9,55 & 564 & 164 & 164 \\
\hline Salomăo et al., 1988 & Brasil, Carajás & $2 * 500 \times 10$ & 10 & 484 & 122 & 122 \\
\hline Gentry, 1988a & Pen, Yanamono & 1ha & 10 & 508 & 283 & 283 \\
\hline Gentry, 1988a & Peru, Mishana & Tha & 10 & 842 & 275 & 275 \\
\hline Gentry, 1988a & Peru, Cocha Cashu & tha & 10 & 650 & 189 & 189 \\
\hline Gentry, 1988a & Peru, Cabeza de Mono & Tha & 10 & 520 & 169 & 169 \\
\hline Gentry, 1988a & Pen, Tambopata & tha & 10 & 585 & 168 & 168 \\
\hline Gentry, 1988a & Brasil / Venezuela & 1ha & 10 & 493 & 89 & 89 \\
\hline Balée, 1987 & Brasil, També & Tha & 10 & 456 & 138 & 138 \\
\hline Baslev etal., 1987 & Equador, Yasuni & QC & 10 & 804 & 244 & 228 \\
\hline Mori \& Boom, 1987 & Gulana Francesa & QC & 10 & 800 & 295 & - \\
\hline Silva et al., 1987 & Brasil. Carajás & $10 \times 1000$ & 9,55 & 456 & 210 & 210 \\
\hline Absy et al., 1986/87 & Brasil, Jarú & $6(10 \times 1000)$ & 10 & 2235 & 278 & $103-136$ \\
\hline Balée, 1986 & Brasil, Maranhăo & $20 \times 500$ & 10 & 498 & 117 & 117 \\
\hline Boom, 1986 & Bolvia, Alto Ivon & $10 \times 1000$ & 10 & 649 & 94 & 94 \\
\hline Campbel etal., 1986 & Brasil , Xingu & $10 \times 3000$ & 10 & 1420 & 265 & $118-133$ \\
\hline Silva et al., 1986 & Brasil, Carajás & $20 \times 500$ & 9,55 & 516 & 125 & 125 \\
\hline Uhl \& Murphy, 1981 & San Carlos, Venezuela & 1 ha & 10 & 744 & 83 & 83 \\
\hline Dantas et al., 1980 & Brasil, Capitáo Poço & $40^{*}(10 \times 25)$ & 9,55 & 504 & 120 & 120 \\
\hline Dantas \& Muller, 1979 & Brasil, Transamazonica & $40 *(10 \times 25)$ & 9,55 & 578 & 101 & 101 \\
\hline Prance et al., 1976 & Brasil, Manaus & $125 \times 80$ & 15 & 350 & 179 & 179 \\
\hline Rodrigues, 1963 & Brasil, Amapá & $11^{*}(10 \times 100)$ & 15 & 347 & & \\
\hline Rodrigues, 1963 & Brasil, Amapá & $15^{*}(10 \times 100)$ & 15 & 307 & 96 & 96 \\
\hline Cain et al., 1956 & Brasil, Belém & $20^{*}(10 \times 100)$ & 10 & 1188 & 173 & 144 \\
\hline Pires et al... 1953 & Brasil, Belém & $3,5 \mathrm{ha}$ & 10 & 1482 & 179 & 108 \\
\hline Black et al., 1950 & Brasil, Belém & $100 \times 100$ & 10 & 423 & 87 & 87 \\
\hline
\end{tabular}

$\mathrm{QC}=$ quadrante centrado, - dado năo disponivel, * número de parcelas 
(Hubbell, 1979; Hubbell \& Foster, 1986; Condit et al., 1996). Florestas tropicais na América Central, Malásia e Índia estão sendo estudadas em parcelas permanentes de $50 \mathrm{ha}$, onde todos os indivíduos com mais de $1 \mathrm{~cm}$ de diâmetro são monitorados (Condit, 1995). Estudos deste porte, concentrando esforços de pesquisa em uma única parcela de grande extensão, podem gerar informações que nos permitam responder questões relevantes quanto à estrutura, dinâmica e diversidade dessas matas e quanto à adequação de metodologias de amostragem. De qualquer modo, só será possível uma visão mais abrangente sobre as comunidades das florestas amazônicas quando forem conciliados estudos a longo prazo em parcelas permanentes a um melhor conhecimento da sistemática e distribuição geográfica das espécies.

\section{AGRADECIMENTOS}

Este estudo faz parte do doutorado realizado no Departamento de Botânica do Instituto de Biociências da Universidade de São Paulo e financiado pela Fundação de Amparo à Pesquisa do Estado de São Paulo (FAPESP). Agradeço ao Dr. José Rubens Pirani e Dr. Scott Mori pelo incentivo e pela revisão do texto em sua primeira versão.

\section{Bibliografia citada}

Absy, M.L.; Prance, G.T.; Barbosa, E.M. 1986/ 1987. Inventário florístico de floresta natural na área da estrada Cuiabá-Porto Velho (BR - 364). Acta Amazonica, 16/ 17(supl.): 85-121.
Almeida, S.S; Lisboa, P.L.B.; Silva, A.S. 1993. Diversidade florística de uma comunidade arbórea na estação científica "Ferreira Pena", em Caxiuanã (Pará). Bol. Mus. Paraense Emilio Goeldi Bot., 9(1): 93128.

Amaral, I.L. 1996. Diversidade floristica em floresta de terra firme, na região de Urucu - AM. Dissertação de Mestrado, Instituto Nacional de Pesquisas da Amazônia (INPA)/Fundação Universidade do Amazônas (FUA). Manaus, Amazonas. falta número de páginas

Ayres, J.M. 1981. Observações sobre a ecologia e o comportamento dos Cuxiús (Chiropotes albinasus e C. satanus). Dissertação de Mestrado. INPA. Manaus. falta número de páginas

Balée, W. 1986. Análise preliminar de inventário florestal e a etnobotânica Ka'apor (Maranhão). Bol. Mus. Paraense Emilio Goeldi, 2(2): 141-167.

Balée, W. 1987. Etnobotânica quantitativa dos indios Tembé (Rio Gurupi, Pará). Bol. Mus. Paraense Emilio Goeldi, 3(1): 29 50.

Balslev, H.; Luteyn, B.; Øllgaard, B.; HolmNielsen, 1987. Composition and structure of adjacent unflooded and floodplain forest in Amazonian Ecuador. Opera Botanica, 92: 37-57.

Bastos, A.M. 1948. As matas de Santa Maria do Vila Nova. Anuário Brasileiro de Economia Florestal I: 281-288.

Bastos, A.M. 1960. A floresta do AmapariMatapi-Cupixi (inventário florestal). Serviço Florestal Boletim, 2. Rio de Janeiro. p54.

Black, G.A.; Dobzhansky, T.H.; Pavan, C. 1950. Some attempts to estimate species diversity and population density of trees in Amazonian forests. Bot. Gaz., 111 (4): 413-425.

Boom, B.M. 1986. A forest inventory in Amazonian Bolivia. Biotropica, 18(4): 413425.

Boom, B.M. 1987. Ethnobotany of the 
Chácobo indians, Beni, Bolivia. Advances Econ. Bot., 4: 1-68.

Bray, J.R.; Curtis, C.T. 1957. An ordination of upland forest communities of southern Wisconsin. Ecol. Monogr, 27: 325-349.

Cain, S.A.; Castro, G.M.O; Pires, J.M; Silva, N.L. 1956. Application of some phytosociological techniques to Brazilian rain forest. Amer. J. Bot., 43: 911-941.

Campbell, D. G.; Daly, D. C.; Prance, G. T.; Maciel, U.N. 1986. Quantitative ecological inventory of terra firme and várzea tropical forest on the Rio Xingú, Brazilian Amazonia, Brittonia, 38(4): 369-393.

Clinebell II, R.R.; Phillips, O.L.; Gentry, A.H.; Stark, N.; Zuuring, H. 1995. Prediction of neotropical tree and liana species richness from soil and climatic data. Biodiversity and Conservation, 4: 56-90.

Comiskey, J.A.; Aymard, G. \& Dallmeier, F. 1994. Structure and composition of lowland mixed forest in the kwani region of Guyana. Bollania 10: 13-28.

Condit, R.; Hubbell, S.P.; Lafrankie, J.V.; Sukumar, R.; Mankaran, N.; Foster, R.B.; Ashton, P. 1996. Species-area and speciesindividual relationships for tropical trees: a comparison of three 50 -ha plots. $J$. Ecol., 84: 549-562.

Condit, R. 1995. Research in large, long-term tropical forest plots. Trends Ecol, and Evol., 10: 18-22.

Cottam, G.; Curtis, J.T. 1956. The use of distance measures in phytosociological sampling. Ecology, 37(3): 451-460.

Curtis, J.T.; Macintosh. 1951. An upland forest continum in the prairie-forest border region of Wisconsin. Ecology, 32: 476496.

Dantas, M.; Muller, N.A.M. 1979. Estudos fito-ecológicos do trópico úmido brasileiro II. Aspectos fitossociológicos de mata sobre latossolo amarelo em Capitão Poço, Pará. Boletim de Pesquisa, 9. Belem, CPATU/EMBRAPA. falta número de páginas

Dantas, M.; Rodrigues, I.A.; Muller, N.A.M. 1980. Estudos fito-ecológicos do trópico úmido brasileiro I. Aspectos fitossociológicos de mata sobre terra roxa na região de Altamira, Pará. Congresso Nacional de Botânica XXX. Campo Grande, MS, Anais da Sociedade Botânica do Brasil. falta número de páginas

Davis, T.A.W.; Richards, P.W. 1933. The vegetation of Moraballi Creek, British Guiana: an ecological study of a limited area of tropical rain forest I. J. Ecol., 21(2): 350-384.

Davis, T.A.W.; Richards, P.W. 1934. The vegetation of Moraballi Creek, British Guiana: an ecological study of a limited area of tropical rain forest II. J, Ecol., 22: 106-155.

Duivenvoorden, J.F. 1995. Tree species composition and rain forest-environment relationships in the middle Caquetá area, Colombia, NW Amazonia. Vegetatio, 120: 91-113.

Duivenvoorden, J,F. 1996. Patterns of tree species richness in rain forests of the middle Caquetá area, Colombia, NW Amazonia. Biotropica, 28(2): 142-158.

Faber-Langendoen, D.; Gentry, A.H. 1991. The structure and diversity of rain forests at Bajo Calima, Chocó region, western Colombia. Biotropica, 23(1): 2-11.

Ferreira, L.V.; Prance, G.T. 1998. Species richness and floristic composition in four hectares in the Jaú National Park in upland forests in Central Amazonian. Biodiversity and Conservation, 7(10): 1349-1364.

Gentry, A. H.; Emmons, L. H. 1987. Geographical variation in fertility, phenology, and composition of the understory of neotropical forests. Biotropica, 19(3): 216-227.

Gentry, A. H. 1982. Patterns of neotropical plant species diversity. Evol. Biol., 15: 184.

Gentry, A. H. 1986. An overview of neotropical phytogeographic patterns with an emphasis on Amazonia. Anais $1^{\prime \prime}$ Simpósio do Trópico Úmido. Brasilia. falta número de páginas

Gentry, A. H. 1988a. Tree species richness of upper Amazonian forests. Procl. Natl. 
Acad. Sci.USA, 85:156-159.

Gentry, A. H. 1988b. Changes in plant community diversity and floristic composition on environmental and geographical gradients. Ann. Missouri. Bot, Gard., 75(1); 134.

Gentry, A. H. 1992. Tropical forest diversity: distributional patterns and their conservational significance. Oikos, 63(1): 19-28.

Glerum, B.B.; Smith, G. 1960. Inventário florestal total na região do rio Curuá-Una (Santarém). Relatório FAO n ${ }^{\circ} 1271$. falta numero de páginas

Glerum, B.B.; Smith, G. 1962. Levantamento combinado floresta-solo ao longo da rodovia BR-14 entre São Miguel e Imperatriz. Relatório $F A O \mathrm{n}^{\circ} 1483$. falta número de páginas

Glerum, B.B. 1960. Levantamento florestal na região entre o rio Caeté e o rio Maracassumé. Relatório FAO n ${ }^{\circ} 1250$. falta número de páginas

Glerum, B.B. 1962a. Levantamento florestal na região de Ucuúba no rio Tocantins. Relatório $F A O \mathrm{n}^{\circ} 1492$. falta número de páginas

Glerum, B.B. 1962b. Levantamento florestal piloto na região de Mogno nos Estados de Goiás e Pará. Relatório $F A O \mathrm{n}^{\circ} 1562$, falta número de páginas

Grubb, P.J.; Lloyd, J.R.; Pennington, T.D.; Whitmore, T.C. 1963. A comparison of montane and lowland rain forest in Ecuador I. The forest structure, physiognomy, and floristics, J. Ecol., 51(3): 567-601.

Heinsdijk, D.; Bastos, A.M. 1965. Inventários florestais na Amazônia. Relatório FAO n ${ }^{\circ}$ 2159. falta número de páginas

Heinsdijk, D. 1957. Levantamento florestal na região entre o rio Tapajós e o rio Xingu. Relatório FAO $\mathrm{n}^{\circ} 601$. falta número de páginas

Heinsdijk, D. 1958a. Levantamento florestal na região entre o rio Xingu e o rio Tocantins. Relatório $F A O \mathrm{n}^{\circ} 949$. falta número de páginas

Heinsdijk, D. 1958b. Levantamento florestal na região entre o rio Madeira e o rio Tapajós. Relatório FAO n ${ }^{\circ} 969$. falta número de páginas

Heinsdijk, D. 1958c. Levantamento florestal na região entre o rio Tocantins e os rios Guamá e Capim. Relatório FAO n 992. falta número de páginas

Heinsdijk, D. 1960. As florestas de terra firme nos solos Terciário e Quaternário ao sul do rio Amazônas. Relatório FAO n 1284. falta número de páginas

Heinsdijk, D. 1964. Amostragem "zero" nos levantamentos florestais. Boletim $n^{\circ} 8$, do Ministério da Agricultura. falta número de páginas

Heinsdijk, D. 1965. A distribuição dos diâmetros nas florestas brasileiras. Boletim $n^{\circ} 11$, do Ministério da Agricultura. falta número de páginas

Heinsdijk, D. 1966. Inventário florestal. Relatório FAO n ${ }^{\circ} 2159$. falta número de páginas

Hubbell, S.P.; Foster, R.B. 1986. Commonness and rarity in a neotropical forest: implications for tropical tree conservation. In: M. Soulé (ed.), Conservation Biology: the science of scarcity and diversity. Sunderland. Massachusetts, falta número de páginas

Hubbell, S.P. 1979. Tree dispersion, abundance, and diversity in a tropical dry forest. Science, 203: 1299-1309.

Korning, J.; Thomsen, K.; Ollgard, B. 1991. Composition and structure of a species rich Amazonian rain forest obtained by two different sampling methods. Nordic J. Bot., 11: 103-111

Lechthaler, R. 1956. Inventário de árvores de um hectare de terra firme. INPA, Botânica, 3. falta número de páginas

Lisboa, P.L.B. 1989. Estudo florístico da vegetação arbórea de uma floresta secundária, em Rondônia. Bol. Mus. Paraense Emilio Goeldi Bot., 5(2): 145162.

Maciel, U.N.; Lisboa, P.L.B. 1989. Estudo florístico de 1 hectare de mata de terra 
firme no $\mathrm{Km} 15$ da rodovia Presidente Médici - Costa Marques (RO-429), Rondônia. Bol. Mus. Paraense Emilio Goeldi Bot., 5(1): 25-37.

Milliken, W. 1998. Structure and composition of one hectare of Central Amazonian terra firme forest. Biotropica, 30(4) falta número de páginas

Milliken, W.; Miller, R. P.; Pollard, S.R.; Wandelli, E. 1992. Ethnobotany of the Waimiri Atroari indians of Brazil. Royal Botanic Gardens. Kew. falta número de paiginas

Morellato, P.L.C.; Rosa, N.A. 1991. Caracterização de alguns tipos de vegetação na região amazônica, Serra dos Carajás, Pará, Brasil. Revta. Brasil. Bot. 14: 1-14.

Mori, S.A.; Boom, B. 1987. The Forest. In: S.A. Mori (org.) The Lecythidaceae of a lowland neotropical florest: La Fumée Mountain, French Guyana. Mem. New York Bot. Gard., 44: 9-29.

Mori, S.A.; Boom, B.M.; Carvalino, A.M.; Santos, T.S. 1983. Ecological importance of Myrtaceae in an eastern Brazilian moist forest. Biotropica, 15: 68-69.

Mori, S.A.; Rabelo, B.V.; Tsou, C.H.; Daly, D. 1989. Composition and structure of an eastern Amazonian forest at Camapai, Amapá, Brasil. Bol. Mus. Paraense Emilio Goeldi, 5(1): 3-18.

Mueller-Dombois, D.; Ellenberg, H. 1974. Aims and methods of vegetation ecology. John Wiley. New York. falta número de páginas

Muniz, F. H.; Cesar, 0,; Monteiro, R. 1994a. Aspectos floristicos quantitativos e comparativos da vegetação arbórea da Reserva Florestal do Sacavém, São Luís, Maranhão (Brasil). Acta Amazonica, 24(3/ 4): $189-218$

Muniz, F. H.; Cesar, 0.; Monteiro, R. 1994b. Fitossociologia da vegetação arbórea da Reserva Florestal do Sacavém, São Luís, Maranhão (Brasil). Acta Amazonica 24(3/ 4): $219-236$

Oliveira, A. A. de; Daly, D. no prelo. Geo- graphic distribution of tree species in the region of Manaus, Brazil: Implications for regional diversity and conservation que revista

Oliveira, A. A. de; Mori, S. A. no prelo. A central Amazonian terra firme forest. I. High tree species richness on poor soils. que revista

Oliveira, A. A. de 1997. Diversidade, estrutura $e$ dinâmica do componente arbóreo de uma floresta de terra firme de Manaus, $A M$. Tese de Doutorado. Instituto de Biociências. Universidade de São Paulo. São Paulo. falta número de páginas

Phillips, O.L.; Hall, P.; Gentry,A.H.; Sawyer, S.A.; Vasquez, R. 1994. Dinamics and species richness of tropical rain forests. Proc. Natl. Acad. Sci. USA falta número de páginas

Pires, J.M.; Prance, G.T. 1977. The Amazon forest: a natural heritage to be preserved. In: G.T. Prance; Elias, T.S. (eds.), Extinction is Forever. New York Botanical Garden. New York.

Pires, J.M.; Dobzhansky, T.H.; Black, G.A. 1953. An estimate of the number of trees in an Amazonian forest community. Bot. Gaz., 114(4): 467-477.

Porto, M.L.; Longhi, H.M.; Citadini, V.; Ramos, R.F.; Mariath, J.E.A. 1976. Levantamento fitossociológico em área de "mata-de-baixio" na estação experimental de silvicultura tropical - INPA - Manaus Amazonas. Acta Amazonica, 6(3): 301318.

Prance, G.T. 1990. The floristic composition of the forests of central Amazonian Brazil. In: A.H. Gentry (ed.). Four Neotropical Rainforests. Yale University Press, New Haven, falta número de páginas

Prance, G.T.; Rodrigues, W.A.; Silva, M.F. 1976. Inventário floristico de um hectare de mata de terra firme $\mathrm{km} 30$ da Estrada Manaus-Itacoatiara. Acta Amazonica, 6(1): 9-35.

Preston, F.W. 1948. The commonness and rarity of species. Ecology, 29(3): 254-283.

RadamBrasil. 1968-1978. Levantamento de 
Recursos Naturais, vols 1-18. Ministério de Minas e Energia. Departamento Nacional de Produção Mineral, Rio de Janeiro, falta número de páginas

Ramos, A.A.; Vieira, A.N.; vivacqua, C.A.; Alencar, J.C.; Barros, J.C.M.; Netto, S.P. 1972. Levantamento florestal do distrito agropecuário da Zona Franca de Manaus. Rev. Floresta, 4(1): 40-53.

Rankin-De-Merona, J.M.; Prance, G.T.; Hutchings, R.W.; Silva, M.F.; Rodrigues, W.A.; Uehling M.E. 1992. Preliminar results of a large-scale tree inventory of upland rain forest in the central Amazon. Acta Amazonica, 22(4): 493-534.

Raunkiaer, C. 1934. The life forms of plants and statistical geography. Clarendon Press. Oxford. falta número de páginas

Richards, P.W. 1952. The tropical rainforest: an ecological study. London. $450 \mathrm{p}$.

Rodrigues, W.A. 1963. Estudo de 2,6 hectares de mata de terra firme da Serra do Navio, território do Amapá. Bol. Mus. Paraense Emilio Goeldi, 19: 1-42.

Rodrigues, W.A. 1967. Inventario florestal piloto ao longo da estrada ManausItacoatiara, Estado do Amazonas: dados preliminares. Atas do Simpóiso Biota Amazônica v. 2 : 257-267.

Rollet, B. 1993. Tree populations in natural tropical rain forest. Bois et Forêsts des Tropiques, 236(2): 43-55.

Salomão, R.P.; Lisboa, P.L.B. 1988. Análise ecológica da vegetação de uma floresta pluvial tropical de terra firme, Rondônia. Bol. Mus. Paraense Emilio Goeldi, 4(2): 195-233.

Salomão, R.P. 1991. Uso de parcela permanente para estudo da vegetação da floresta tropical úmida. I. Município de Marabá, Pará. Bol. Mus. Paraense Emilio Goeldi, 7(2) 543-604.

Salomão, R.P.; Silva, M.F.F.; Rosa, N.A. 1988. Inventário ecológico em floresta pluvial tropical de terra firme, Serra Norte,
Carajäs, Pará. Bol. Mus. Paraense Emilio Goeldi, 4(1): 1-46.

Silva, A.S.L; Lisboa, P.L.B.; Maciel, U.N. 1992. Diversidade florística e estrutura em floresta densa da bacia do Rio Juruá. Bol. Mus. Paraense Emilio Goeldi, 8(2): 203-258.

Silva, M.F.; Rosa, N.A. 1989. Análise do estrato arboreo da vegetação sobre jazidas de cobre na Serra dos Carajás - PA. Bol. Mus. Paraense Emilio Goeldi, 5(2): 175205.

Silva, M.F,; Rosa, N.A.; Oliveira, J. 1986. Estudo botânico na área do projeto ferro Carajás. 3. Aspectos florísticos da mata do aeroporto da Serra Norte - PA. Bol. Mus. Paraense Emilio Goeldi, 2(2): 169-187.

Silva, M.F.; Rosa, N.A.; Oliveira, J. 1987. Estudo botânico na área do projeto ferro Carajás. 5. Aspectos florísticos da mata do rio gelado, Pará. Bol. Mus. Paraense Emilio Goeldi, 3(1): 1-20.

Spichiger, R.; P.-A. Loizeau; Latour, C.; Barriera, G. 1996. Tree species richness of a South-Western Amazonian forest (Jenaro Herrera, Peru, $\left.73^{\circ} 40^{\prime} \mathrm{W} / 4^{0} 54^{\prime} \mathrm{S}\right)$. Candollea, 51: 559-577.

Steege, H.S. 1998. The use of forest inventory data for a National Protected Area Strategy in Guyana. Biod. Conserv. 7 (II): 1457-1483.

SUDAM. 1974. Levantamentos florestais realizados pela missão $F A O$ na Amazônia. Belém, v. 2. falta número de páginas

Takeuchi, M. 1960. A estrutura da vegetação na Amazônia I - A mata pluvia tropical. Bol. Mus. Paraense Emilio Goeldi, 6: 1 29.

Takeuchi, M. 1961. The structure of the Amazonian vegetation II. Tropical rain forest. J. Fac. Sci. Univ. Tokyo III, 8(1): 1-26.

Takeuchi, M. 1962. The structure of the Ama-

\section{Aceito para publicação em 11/10/2000}


zonian vegetation $\mathrm{V}$. Tropical rain forest near Uaupés. J.Fac. Sci. Univ. Tokyo III, 8(4-7): 289-296.

Terborgh, J.; Andresen, E. 1998. The compoistion of Amazonian forests: patterns at local and regional scales. J. Trop. Ecol.,14: 645-664.

Thompson, J.; Proctor, J.; Viana, V.; Milliken, W.; Ratter, J.A.; Scott, D.A. 1992. Ecological studies on a lowland evergreen rain forest on Maracá Island, Roraima, Brazil. I. Physical environment, forest structure and leaf chemistry. J. Ecol., 80: 689-703

Uhl, C.; Murphy, P.G. 1981. Composition, structure, and regeneration of terra firme in the Amazon basin of Venezuela. Tropic. Ecol., 22(2): 219-237.

Valencia, R.; Balslev, H.; Paz Y Mino, G. C. 1994. High tree alpha-diversity in Amazonian Ecuador. Biodiv. Conserv., 3: 2128. 